\title{
UTILISATION COMPARÉE DE L'ENSILAGE DE MAÏS ET DE L'ENSILAGE D'HERBE POUR LA PRODUCTION LAITIÈRE
}

\author{
R. VÉRITÉ et M. JOURNET \\ avec la collaboration technique de Jeanne FLÉchet, J. LEFAIVRe, \\ Renée Lifiatvre, 13. Marquis et A. Ollier \\ Station de Recherches sur l'Élevage des Ruminants, \\ Centre de Recherches de Clermont-Ferrand, I. N. R. A., \\ 63 - Saint-Genès-Champanelle
}

\section{RÉSUMÉ}

Le but de l'essai a été de comparer l'utilisation de l'ensilage de maïs et de l'ensilage d'herbe de prairie naturelle pour la production laitière et de connaître en particulier leur effet sur l'orientation du métabolisme énergétique des vaches.

Pour cela, après une période pré-expérimentale de 3 semaines, nous avons distribué, à 2 lots de 6 vaches, 2 rations isoénergétiques et isoazotées à base l'une d'ensilage d'herbe ("Iot herbe ") et l'autre d'ensilage de maïs (" lot maïs ") et contenant toutes deux des betteraves sucrières fraîches (Io premières semaines) ou déshydratées (7 dernières semaines). La quantité d'aliment concentré distribuéc à chaque vache était calculée d'après sa production de lait en période préexpérimentale et une décroissance théorique de cette production de I,5 p. 1oo par semaine. La digestibilité de chaque ensilage mesurée avec un lot de 4 moutons et un lot de 4 vaches (tabl. I) a servi de base au calcul de la valeur énergétique.

La production de lait des animaux du "lot herbe " a été plus élevée que celle des animaux du " lot maïs" (+ I,25 kg; $P<0,05)$ mais leur gain de poids vif a été plus faible (- roo ou - I8o g par jour suivant la méthode d'estimation ; $\mathrm{P}<0,05)$. Les taux butyreux et les productions de matières grasses et de matières azotées n'ont pas été significativement différents ; par contre, le taux de matières azotées du lait a été plus élevé dans le "lot maïs" $(+2,4 \mathrm{~g} \mathrm{p}$. I ooo ; $\mathrm{P}<0,05$ ) (tabl. 2, fig. I).

Ces résultats ont été interprétés en fonction : Io de la précision de l'estimation des apports alimentaires ; $2^{\circ}$ de la composition du mélange d'acides gras volatils présent dans le rumen (la proportion d'acide acétique a été plus faible et celle d'acide butyrique plus élevée avec le « lot maïs " qu'avec le "lot herbe " : 49 p. I oo contre 57 p. Ioo et 27 p. Ioo contre I9 p. roo, (tabl. 6) ; $3^{\circ}$ de la composition en acides gras des matièrcs grasses du lait (tabl. 5); $4^{\circ}$ de la teneur du sang en corps cétoniques et de leur nature.

Un effet d'associativité entre aliments a été observé (tabl. 3 et fig. I) puisque le remplacement des betteraves fraîches par des betteraves déshydratées a entraîné une baisse importante du taux butyreux (- 3,4 à - I $4,4 \mathrm{~g}$ p. I ooo) ainsi qu'une modification de la composition en acides gras du lait (tabl. 5) dans le lot maîs seulement. Ces changements ont été reliés à la diminution de la proportion d'acide butyrique dans le rumen (tabl. 6) alors que celle d'acide propionique ne variait pas. 


\section{INTRODUC'TION}

L'énergie métabolisable ingérée par une vache laitière est utilisée, une fois les besoins d'entretien couverts, pour la production du lait et pour la constitution de réserves corporelles. Le partage entre ces deux utilisations dépend du niveau énergétique de la ration (loi des rendements décroissants), du potentiel laitier des animaux, du stade de lactation (BROSTER, I960) et de 1'histoire nutritionnelle de la vache. I a nature de la ration a probablement un effet sur ce partage puisque, même dans le cas d'une alimentation globalement équilibrée, elle commande les fermentations dans le rumen et donc les métabolites disponibles au niveau sanguin. D'autre part, elle peut orienter l'activité enzymatique des différents tissus (OPSTVLNT et al., I967). BIAXTIER (I962) a montré que le rendement de l'énergie métabolisable pour la production laitière seule variait avec la composition du mélange d'AGV dans le rumen. FLA'TT et al (I969) ont également montré (que ce rendement variait avec le type de ration. ØRskov et al. (I969) dans des essais de bilan, ont comparé 3 régimes isoénergétiques comprenant des infusions intra-ruminales d'acide acétique ou d'acide propionique. L'efficacité globale de l'énergie a été la même pour les 3 régimes mais le partage de l'énergie entre la production de lait et le dépôt de graisse corporelle a nettement varié d'un lot à l'autre.

L'influence du type de ration sur l'utilisation de l'énergie a été nettement mise en évidence dans quelques cas particuliers. On sait ainsi (VAN SoEST, I965 ; JoRGENSEN, I 965 ; RÉMOND, non publié) que les rations à base de fourrages broyés agglomérés ou d'aliments concentrés font diminuer le taux butyreux et parfois même la production laitière mais favorisent le dépôt de tissus corporels. Par ailleurs, il semble que l'ensilage d'herbe non préfané favorise plus la production laitière que le foin obtenu à partir des mêmes plantes (cf. revues de BrEIREM (I965), de MERriL et SLACK (I965) et de LABADIE (I968)).

Les vaches laitières recevant de l'ensilage de maïs ont tendance à engraisser, probablement en partie par suite du niveau énergétique élevé de la ration, bien que la persistance de la production laitière ne soit que normale, voire faible. Il était donc intéressant de comparer, avec des vaches laitières, deux régimes isoénergétiques l'un à base d'ensilage d'herbe et l'autre à base d'ensilage de maïs, pour déceler d'éventuelles différences dans l'utilisation de l'énergie.

\section{MATÉRIEL, ET MÉTHODES}

\section{Animaux}

Dix-huit vaches (Frisonnes Pie Noire) en $\mathrm{I}^{\mathrm{re}}, 2^{\mathrm{e}}$ ou $3^{\mathrm{c}}$ lactation, vêlées depuis $\mathrm{I}$ à 3 mois ont été appariées 2 à 2 suivant le numéro de lactation et la production laitière. Trois vaches ayant été éliminées en cours d'expériences (mammite, boiterie,...), l'expérience s'est terminée avec seulement 6 paires d'animaux. Les vaches ont été maintenues en permanence en stabulation entravée sur litière de copeaux. 


\section{Alimentation}

Au cours de la période pré-expérimentale de 3 semaines, les vaches ont reçu un régime commun de foin (cn moyenne $3,7 \mathrm{~kg}$ de matière sèche (MS)), d'ensilage de maîs (en moyenne $4,3 \mathrm{~kg}$ MS), de betteraves sucrières fraîches (3 kg MS) et d'aliment concentré dont la quantité (5,3 kg MS en moyenne) était calculée pour couvrir les besoins. L'appariement des vaches a été fait à partir des résultats des 2 dernières semaines de cette période. La production laitière, le taux butyreux, le taux de matières azotées et lo poids vif étaient alors respectivement pour les lots I et II

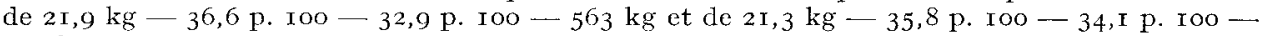
$579 \mathrm{~kg}$.

Après une semaine de transition, la période expérimentale a duré I 7 semaines. Le lot I ("lot herbe ") a reçu de l'ensilage direct (avec mélasse) de prairie naturelle à volonté (24 p. Ioo MS), Io $\mathrm{kg}$ de betteraves sucrières, de l'aliment concentré et du tourteau d'arachide. Dans Ie lot II (" lot maïs ), l'ensilage de maïs (en quantité limitée) (24 p. Ioo MS) remplaçait (quantitativement sur la base de l'énergie nette pour chaque paire de vaches) l'ensilage d'herbe. Les quantités d'aliment concentré et de tourteau distribuées ont été ajustées chaque semaine pour chaque vache de façon à couvrir les besoins de lcur production théorique. Celle-ci était obtenue en appliquant à la production de référence (moyenne pré-expérimentale) un coefficient de décroissance hebdomadaire de I p. Ioo en $I^{\text {re }}$ lactation et $\mathrm{I}, 5 \mathrm{p}$. Ioo en $2^{\mathrm{e}}$ ou $3^{\mathrm{e}}$ lactation. L'horaire de distribution était le suivant : 8 hcures (aliment concentré + betteraves), 9 heures (ensilage), I 7 heures (aliment concentré), I 8 heures (ensilage). Au cours de la no semaine expérimentale, Ies cossettes fraîches de betteraves sucrières ont été remplacées par des cossettes déshydratées. De l'urée avait été ajoutée à l'ensilage de maïs (à raison de $4 \mathrm{~kg}$ par tonne de produit frais) lors de la mise en silo.

Pendant une semaine après la période expérimentale la digestibilité des 2 rations a été mesurée sur 4 vaches de chaque lot par collecte totale des fèces pendant 6 jours.

Au cours de la période post-expérimentale de 3 semaines (dont une semaine de transition), les vaches ont été au pâturage ( $\mathrm{I}{ }^{\mathrm{er}}$ cycle de prairie naturelle) et ont reçu I $\mathrm{kg}$ d'aliment concentré par $2 \mathrm{~kg}$ de lait au-dessus de $\mathrm{I}_{4} \mathrm{~kg}$.

\section{Mesures}

Pour chaque animal nous avons effectué les mesures suivantes:

- quantités journalières offertes et refusées de chaque aliment ;

- quantité de lait produite chaque jour ;

- teneur journalière du lait en matière grasse (Gerber) ;

- teneur hebdomadaire du lait en matières azotées (noir amido) sur un échantillon moyen de 4 traites ( 2 jours consécutifs);

- poids vif des animaux par pesée simple toutes les semaines et pesées 3 jours consécutifs au début et à la fin de l'expérience et pendant la période post-expérimentale ;

- composition en acides gras des matières grasses du lait (par chromatographie en phase gazeuse, DECAEN et ADDA, I966) sur 2 échantillons de lait prélevés au cours de la $7^{\mathrm{e}}$ et de la I $7^{\text {e }}$ semaine ;

- composition du mélange d'A.G.V. dans le jus de rumen (par chromatographie en phase gazeuse, Rigaud et Journet, I970) sur 2 échantillons prélevés par tubage (à II heures) au cours de la $7^{\mathrm{e}}$ et de la $I 7^{\mathrm{e}}$ semaine.

- teneur du sang jugulaire en corps cétoniques (Procos, I96I), (prélèvement à II heures une fois au cours de la $17^{\mathrm{e}}$ semaine).

La teneur en MS de chaque ensilage a été déterminée à l'étuve à $80^{\circ} \mathrm{C}$ chaque jour. Un échantillon moyen a été prélevé au cours des I 7 semaines expérimentales et conservé à - $\mathrm{I}^{\circ} \mathrm{C}$ avant analyse. La digestibilité de chaque ensilage a été mesurée sur un lot de 4 moutons par collecte totale des fèces pendant 7 jours (DEMARquilly et JARRIGE, I970).

\section{Vaches fistulisées}

Deux vaches en lactation, porteuses d'une fistule du rumen, ont reçu successivement la ration du lot I (4 semaines), puis celle du lot II (4 semaines). Deux autres vaches fistulisées ont suivi la séquence inverse. Les betteraves étaient sous forme déshydratée.

Sur chaque animal, les mesures suivantes ont été effectuées : quantités ingérées, comportement alimentaire (RUскевUsch, I963), poids frais et sec des contenus rumino-réticulaires par vidage manuel 3 heures après le repas d'ensilage, composition du mélange d'A.G.V. du rumen (étude cinétique de 39 prélèvements en 24 heures). 


\section{RÉSULTATS}

\section{Valeur nutritive des ensilages}

Les deux ensilages se caractérisent par leur forte teneur en acide lactique (voir tab1. I) et leur faible teneur en acide butyrique ( $<0$, I p. Ioo MS); cette dernière a cependant augmenté en fin d'expérience (juin). Ia digestibilité de la matière organique de l'ensilage de maîs a été la même avec les moutons et les vaches, par contre, celle de l'ensilage d'herbe a été plus élevée $(+2,9$ points) avec les moutons qu'avec les vaches. La valeur énergétique de chaque ensilage a été évaluée (tabl. I) à partir de la teneur en matière organique de l'échantillon moyen et de la digestibilité de la matière organique mesurée avec les moutons (qui est une mesure directe contrairement à celle obtenue avec les vaches) et en utilisant la formule de BrEIREM (I 954) .

\section{TABLEAU I}

Composition, digestibilité et valeur énergétique des ensilages

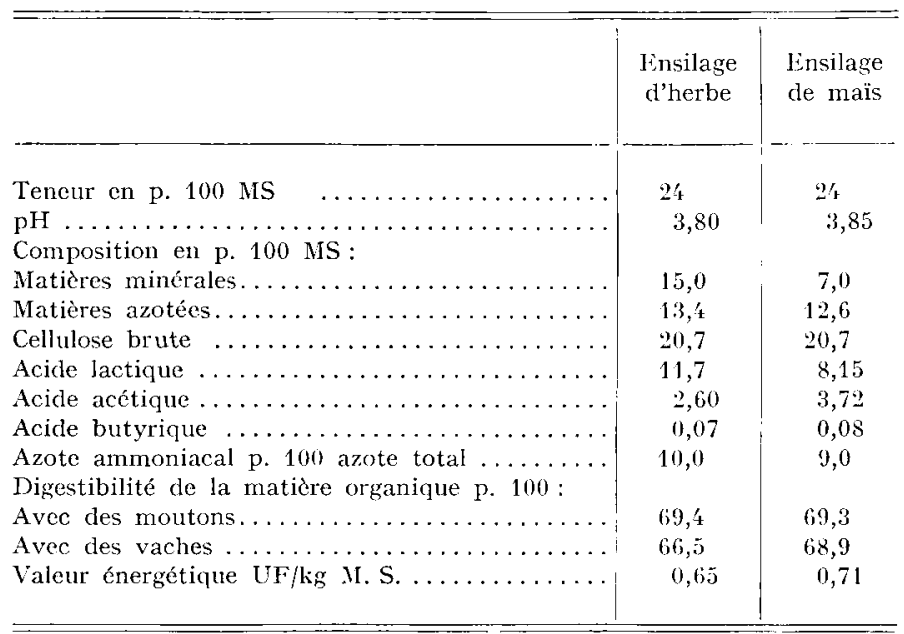

\section{Quantités ingérées et comportement alimentaire}

L,es quantités ingérées d'ensilage d'herbe distribuée à volonté ont été en moyenne de I,53 $\mathrm{kg}$ MS par Ioo $\mathrm{kg}$ de poids vif et ont varié de I,39 à I,82 suivant les animaux. Elles ont d'abord augmenté jusqu'à la $5^{\mathrm{e}}$ semaine ( $\mathrm{r}, 77 \mathrm{p}$. roo), puis diminué ensuite régulièrement jusqu'à la dernière (I,34 p. Ioo). Respectivement pour les lots "herbe " et " maïs ", le niveau d'ingestion de la ration d'ensilage + betterave a été de ro,9 et ro,o $\mathrm{kg}$ MS et celui de la ration totale de I5,0 et I3,9 $\mathrm{kg}$ MS (tab1. 2).

Les apports moyens d'énergie ont été identiques aux besoins calculés à partir de la production théorique pour les animaux du lot "mais " tandis qu'ils dépassaient ces besoins de $0,25 \mathrm{UF}$ pour le lot " herbe ». En fait, comme la production réelle a été inférieure à la production théorique, la suralimentation réelle a été la même dans les 
2 lots : I,o UF pour le lot " maïs » et o,9 UF pour le lot " herbe ". L'apport azoté des animaux du lot " maïs" a été plus faible que celui des animaux du lot " herbe " mais cependant suffisant pour couvrir les besoins ( $\left.{ }^{1}\right)$ d'entretien et de production du lait. Dans le lot " maïs ", I 8 p. roo de l'apport azoté était sous forme d'urée dans l'ensilage (I28 g d'urée).

\section{TABLEAU 2}

Quantités ingérées et productions journalières au cours de la période expérimentale

\begin{tabular}{|c|c|c|c|}
\hline & Lot " herbe" & Lot " maïs" & $\begin{array}{c}\text { Seuil de } \\
\text { signification }\end{array}$ \\
\hline 1. Quantités ingérées par animal et par jour & & & \\
\hline Ration totale ( $\mathrm{kg}$ MS) & 15,03 & 13,90 & \\
\hline 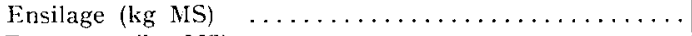 & 8,59 & 7,66 & \\
\hline 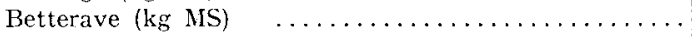 & 2,29 & 2,30 & \\
\hline Tourteau d'arachide (kg MS) $\ldots \ldots \ldots \ldots \ldots \ldots$ & 0,25 & 0,25 & \\
\hline Aliment concentré (kg MS) $\ldots \ldots \ldots \ldots \ldots \ldots \ldots$ & 3,93 & 3,69 & \\
\hline Énergie nette (UF) $\quad \ldots \ldots \ldots \ldots \ldots \ldots \ldots \ldots \ldots$ & 11,75 & 11,38 & \\
\hline $\begin{array}{c}\text { Matières azotées totales }(\mathrm{g}) \ldots \ldots \ldots \ldots \ldots \ldots \ldots \ldots \\
\qquad . \text { Productions journalières }\end{array}$ & $210^{\prime}$ & 1877 & \\
\hline 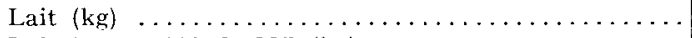 & 17,8 & 16,1 & $5 \%$ \\
\hline Lait à $/ \mathbf{p}$ p. 100 de $\operatorname{MG}(\mathrm{kg}) \quad \ldots \ldots \ldots \ldots \ldots \ldots$ & 17,2 & 15,8 & NS \\
\hline Taux butyreux $(p .1000) \ldots \ldots \ldots \ldots \ldots \ldots$ & 39,1 & 39,1 & NS \\
\hline Taux de protcines $(p .1000) \ldots \ldots \ldots \ldots \ldots \ldots \ldots$ & 30,9 & $3 ', 3$ & $5 \%$ \\
\hline Matières grasses $(g) \ldots \ldots \ldots \ldots \ldots \ldots \ldots$ & 679 & 630 & NS \\
\hline Matières azotées $(g) \ldots \ldots \ldots \ldots \ldots \ldots \ldots \ldots \ldots \ldots$ & $5 / 1$ & $5 \prime 19$ & NS \\
\hline $\begin{array}{l}\text { Coefficient de persistance mensuel de la production de } \\
\text { lait (p. 100) } \ldots \ldots \ldots \ldots \ldots \ldots \ldots \ldots \ldots \ldots \ldots \ldots \ldots \ldots \ldots\end{array}$ & 92,1 & 90,1 & \\
\hline Coefficient de persistance mensuel de la production de & & & \\
\hline lait à 4 p. 100 de $M G(p .100) \quad \ldots \ldots \ldots \ldots \ldots$ & $9: 2,1$ & 88,1 & \\
\hline Poids vif moyen $(\mathrm{kg}) \ldots \ldots \ldots \ldots \ldots \ldots \ldots \ldots \ldots$ & 561 & 572 & \\
\hline Gain de poids vif journalier $(\mathrm{g})$ & & & \\
\hline a) A partir des pesées hebdomadaires $\ldots \ldots \ldots \ldots$ & +80 & +18.2 & $2 \%$ \\
\hline b) A partir des poids en début et fin d'expérience.. & -10 & $+133^{\prime}$ & $5 \%$ \\
\hline
\end{tabular}

Le comportement alimentaire n'a été enregistré que sur les vaches fistulisées (tabl. 3). Il n'y a pas de différence entre les deux types d'alimentation. Cependant, il faut noter que la durée de consommation est courte : 5 h $47^{\prime}$ pour l'ensilage d'herbe et 5 h I $5^{\prime}$ pour l'ensilage de maïs.

\section{Production et composition du lait}

I,es vaches du lot « herbe » ont produit en moyenne $\mathrm{I}, 7 \mathrm{~kg}$ de lait de plus par jour que celles du lot " maïs " (tab1. 2). Cette différence, qui n'est plus que de I,25 kg si on tient compte par une analyse de covariance (SNEDECOR, I967) du niveau de la production de référence est cependant significative $(P<0,05)$. L'écart entre les 
2 lots qui était de $0,6 \mathrm{~kg}$ au cours de la période pré-expérimentale s'est accru régulièrement jusqu'à 2,2 en fin d'expérience (3 dernières semaines). La persistance mensuelle de la production laitière a été légèrement meilleure pour le lot " herbe " $(92, \mathrm{I}$ contre 90,I). A la mise à l'herbe, la production du lot "herbe " a augmenté de I,4 kg tandis que celle du lot "mais " augmentait de I,85 kg.

TABLEAU 3

Comportement alimentaire des vaches fistulisécs

(moyenne de 3 vaches)

\begin{tabular}{|c|c|c|}
\hline & Lot " herbe * & Jot " maïs" \\
\hline 1. Quantités ingêrées & & \\
\hline Ration totale (kg M. S.).......... & 12,39 & 12,51 \\
\hline $\begin{array}{l}\text { Proportion d'aliment concentré (p. } 100) \text {.. } \\
\text { 2. Emploi du temps journalier }\end{array}$ & 36 & 33 \\
\hline Consommation (p. 100 du temps total) .. & 21,1 & 21,9 \\
\hline $\begin{array}{l}\text { Rumination (p. } 100 \text { du temps total) ..... } \\
\text { 3. Durée de mastication }\end{array}$ & 33,5 & 35,8 \\
\hline Consommation ( $\mathrm{mn} / \mathrm{kg}$ MS ingćré) .. & 27,4 & 26,1 \\
\hline Rumination $(\mathrm{mn} / \mathrm{kg}$ IS ingéré $) \ldots \ldots$ & 38,8 & 42,9 \\
\hline
\end{tabular}

En moyenne sur les $I 7$ semaines, le taux butyreux et la production de matières grasses n'ont pas été significativement différents entre les 2 lots bien que leurs évolutions aient été différentes (fig. I, tabl. 4) et semblent indiquer l'existence de deux phases dans l'expérience. Au cours de la première période (Io premières semaines) le taux butyreux augmente dans les 2 lots ; cependant par rapport à la période préexpérimentale, l'augmentation est nettement plus forte avec le lot " maïs " $\left(+5,3\right.$ points) qu'avec le lot " herbe " ( + I,4 points). Pendant la $2^{\mathrm{e}}$ période $(7$ dernières semaines) le taux butyreux reste constant dans le lot "herbe " mais baisse considérablement dans le lot "maïs ) : - 8,4 points (de - 3,5 à - I 4,4 suivant les animaux) entre la $10^{\mathrm{e}}$ et la $\mathrm{I}^{\mathrm{e}}$ semaine. Le début de cette baisse se situe vers la Io $^{\mathbf{e}}$ ou I I $^{\mathbf{e}}$ semaine, au moment du remplacement des betteraves sucrières fraîches par des betteraves sucrières déshydratées. Inversement, à la mise à 1'herbe, le taux butyreux baisse de 8,4 points pour le lot " herbe " et de 2,0 points pour le lot " maïs".

La composition des matières grasses (tabl. 5) du lait au cours de la $7^{\mathrm{e}}$ semaine, les vaches recevant alors les betteraves fraîches, a présenté des différences significatives entre les 2 lots : la part des acides courts (jusqu'à l'acide laurique) et celle de l'acide myristique sont significativement plus élevées dans le lot "mais " que dans le lot " herbe ": respectivement I9,4 p. Ioo contre I5,4 p. IOO $(\mathrm{P}<0,05)$ et I5,3 contre I3,O p. IOO ( $\mathrm{P}<0,0 \mathrm{I}$ ) et celle des acides longs (à partir de l'acide stéarique) est significativement plus faible : 25,4 p. Ioo contre 32 , I p. IOo $(\mathrm{P}<0,0$ I). Par contre, la différence de composition n'est pas significative au cours de la $I 7^{\mathrm{e}}$ semaine lorsque les vaches recevaient des cossettes de betteraves déshydratées. La quantité 
TABI,EAU 4

Évolution des productions

\begin{tabular}{|c|c|c|c|c|c|c|c|c|}
\hline & \multirow{2}{*}{\multicolumn{2}{|c|}{$\begin{array}{l}\text { Période pré- } \\
\text { expérimentale }\end{array}$}} & \multicolumn{4}{|c|}{ Période expérimentale } & \multirow{2}{*}{\multicolumn{2}{|c|}{$\begin{array}{l}\text { Période post- } \\
\text { expérimentale }\end{array}$}} \\
\hline & & & \multicolumn{2}{|c|}{$\begin{array}{c}\text { Betteraves } \\
\text { fraîches } \\
\text { (10 semaines) }\end{array}$} & \multicolumn{2}{|c|}{$\begin{array}{l}\text { Betteraves } \\
\text { déshydratées } \\
\text { (7 semaines) }\end{array}$} & & \\
\hline & Herbe & Mais & Herbe & Maïs & Herbe & Maïs & Herbe & Maïs \\
\hline Production de lait $(\mathrm{kg})$ & 21,9 & 21,3 & 18,7 & 17,2 & 16,55 & 14,4 & 17,0 & 14,9 \\
\hline Matières grasses $(g) \ldots$ & 791 & 758 & 710 & 708 & 631. & 517 & 502 & 496 \\
\hline Taux butyreux (p. 1000$) \ldots$ & 36,6 & 35,8 & 38,0 & 41,1 & 38,1 & 35,9 & 29,7 & 33,8 \\
\hline Matières azotées $(\mathrm{g}) \ldots \ldots \ldots$ & 720 & 726 & 570 & 586 & 509 & 495 & 554 & 526 \\
\hline Taux de protéiries (p. 1000 ) & 32,9 & 34,1 & 30,6 & 34,0 & 30,7 & $34, \ell_{k}$ & 32,6 & 35,3 \\
\hline
\end{tabular}

TABIEAU 5

Composition en acides gras des matières grasses du lait (pourcentage sous forme d'esters méthyliques)

\begin{tabular}{|c|c|c|c|c|}
\hline & \multicolumn{2}{|c|}{ Lot " herbe" } & \multicolumn{2}{|c|}{ Lot " maïs" } \\
\hline & $7^{e}$ semaine & $17^{\mathrm{e}}$ semaine & $7^{\mathrm{e}}$ semaine & $17^{\mathrm{e}}$ semaine \\
\hline Production de lait $(\mathrm{kg}) \ldots$. & 17,9 & 15,4 & 16,3 & 13,3 \\
\hline Taux butyreux (p. 1000$) \ldots$ & 38,7 & 40,0 & 40,5 & 34,9 \\
\hline $\begin{array}{l}\text { Composition en poids d'esters } \\
\text { méthyliques (p. 100) : }\end{array}$ & & & & \\
\hline $\mathrm{C}_{4} \ldots \ldots \ldots \ldots \ldots \ldots \ldots$ & 3,9 & 5,5 & 4,4 & 4,3 \\
\hline $\mathrm{C}_{\mathrm{g}} \ldots \ldots \ldots \ldots \ldots \ldots \ldots \ldots \ldots$ & 2,5 & 3,0 & 2,8 & 2,7 \\
\hline $\mathrm{C}_{8} \ldots \ldots \ldots \ldots \ldots \ldots \ldots$ & 1,5 & 1,7 & 1,7 & 1,4 \\
\hline $\mathrm{C}_{10} \ldots \ldots \ldots \ldots \ldots \ldots$ & 2,8 & 3,3 & 3,7 & 2,8 \\
\hline $\mathrm{C}_{11} \ldots \ldots \ldots \ldots \ldots \ldots \ldots$ & 0,5 & 0,4 & 0,6 & 0,5 \\
\hline$c_{12}^{11} \ldots \ldots \ldots \ldots \ldots \ldots \ldots$ & 4,1 & 4,5 & 5,7 & 4,3 \\
\hline $\mathrm{C}_{14}^{14} \ldots \ldots \ldots \ldots \ldots \ldots$ & 11,0 & 11,8 & 13,2 & 11,7 \\
\hline $\mathrm{C}_{14}^{14}: 1, \ldots \ldots \ldots \ldots \ldots$ & 2,0 & 1,7 & 2,2 & 2,4 \\
\hline $\mathrm{C}_{15} \ldots \ldots \ldots \ldots \ldots \ldots \ldots$ & 1,5 & 1,1 & 1,7 & 1,4 \\
\hline $\mathrm{C}_{15: 1} \ldots \ldots \ldots \ldots \ldots \ldots$ & $0, \mathbf{r}_{\mathbf{1}}$ & 0,3 & 0,5 & 0,3 \\
\hline$C_{18} \ldots \ldots \ldots \ldots \ldots \ldots \ldots$ & 31,8 & 32,8 & 31,8 & 33,0 \\
\hline$C_{16}^{10}:{ }_{1} \ldots \ldots \ldots \ldots \ldots$ & 3,4 & 3,5 & 3,8 & 6,0 \\
\hline $\mathrm{C}_{17} \ldots \ldots \ldots \ldots \ldots \ldots \ldots$ & 1,6 & 1,2 & 1,5 & 1,4 \\
\hline$C_{17}:{ }_{1} \ldots \ldots \ldots \ldots \ldots$ & 0,8 & 0,6 & 0,6 & 0,6 \\
\hline$C_{18} \ldots \ldots \ldots \ldots \ldots \ldots \ldots$ & 7,6 & 7,0 & 6,0 & 1,2 \\
\hline $\mathrm{C}_{18}:{ }_{1} \ldots \ldots \ldots \ldots \ldots$ & 18,3 & 16,6 & 15,3 & 18,1 \\
\hline $\mathrm{C}_{18}:{ }_{2} \ldots \ldots \ldots \ldots \ldots \ldots \ldots$ & 3,0 & $2,{ }^{\prime}$ & 2,7 & 2,8 \\
\hline $\mathrm{C}_{18}: 3 \ldots \ldots \ldots \ldots \ldots$ & 1,6 & 1,2 & 0,9 & 0,8 \\
\hline $\mathrm{C}_{20} \ldots \ldots \ldots \ldots \ldots \ldots \ldots$ & 1,6 & 1,2 & 1,0 & 1,3 \\
\hline Rapport $\mathrm{C}_{16: 1} / \mathrm{C}_{16}: 0 \ldots$ & 0,11 & 0,11 & 0,12 & 0,18 \\
\hline Rapport $\mathrm{C}_{18:{ }_{1} / \mathrm{C}_{18}: 0 \ldots}$ & 2,15 & 2,39 & 2,57 & $\dot{4}, 94$ \\
\hline
\end{tabular}


totale d'acides gras longs $\left(>\mathrm{C}_{18}\right)$ sécrétés a été plus élevée $(\mathrm{P}<0,05)$ avec le lot " herbe " qu'avec le lot " maïs " : + 40 et $+43 \mathrm{~g}$ par jour respectivement à la $7^{\mathbf{e}}$ et $\mathbf{I} 7^{\mathbf{e}}$ semaine.

Entre la $7^{\mathrm{e}}$ et la $\mathrm{I}^{\mathrm{e}}$ semaine, la proportion d'acides gras courts $\left(\mathrm{C}_{4} \quad \mathrm{a} \quad \mathrm{C}_{12}\right)$ a augmenté dans le lot " herbe " tandis que dans le lot " maïs" (en particulier pour les 2 vaches ayant la plus forte baisse de taux butyreux) elle diminuait ainsi que celles d'acide laurique et d'acide stéarique. Les deux rapports $\frac{\text { acides gras mono-insaturés }}{\text { acides gras saturés }}$ pour les acides à 16 et I 8 atomes de carbone n'ont pas varié dans le lot " herbe " mais ont augmenté dans le lot " maïs " respectivement de 52 et 92 p. Ioo.

Le taux de matières azotées du lait a été significativement plus élevé $(\mathrm{P}<0,05)$ avec l'ensilage de maîs qu'avec l'ensilage d'herbe. Dans le lot " mais ", il est resté constant et élevé (fig. I), alors que dans le lot " herbe ", il a baissé de plus de deux points au début de l'expérience et n'est revenu à son niveau initial qu'à la mise à
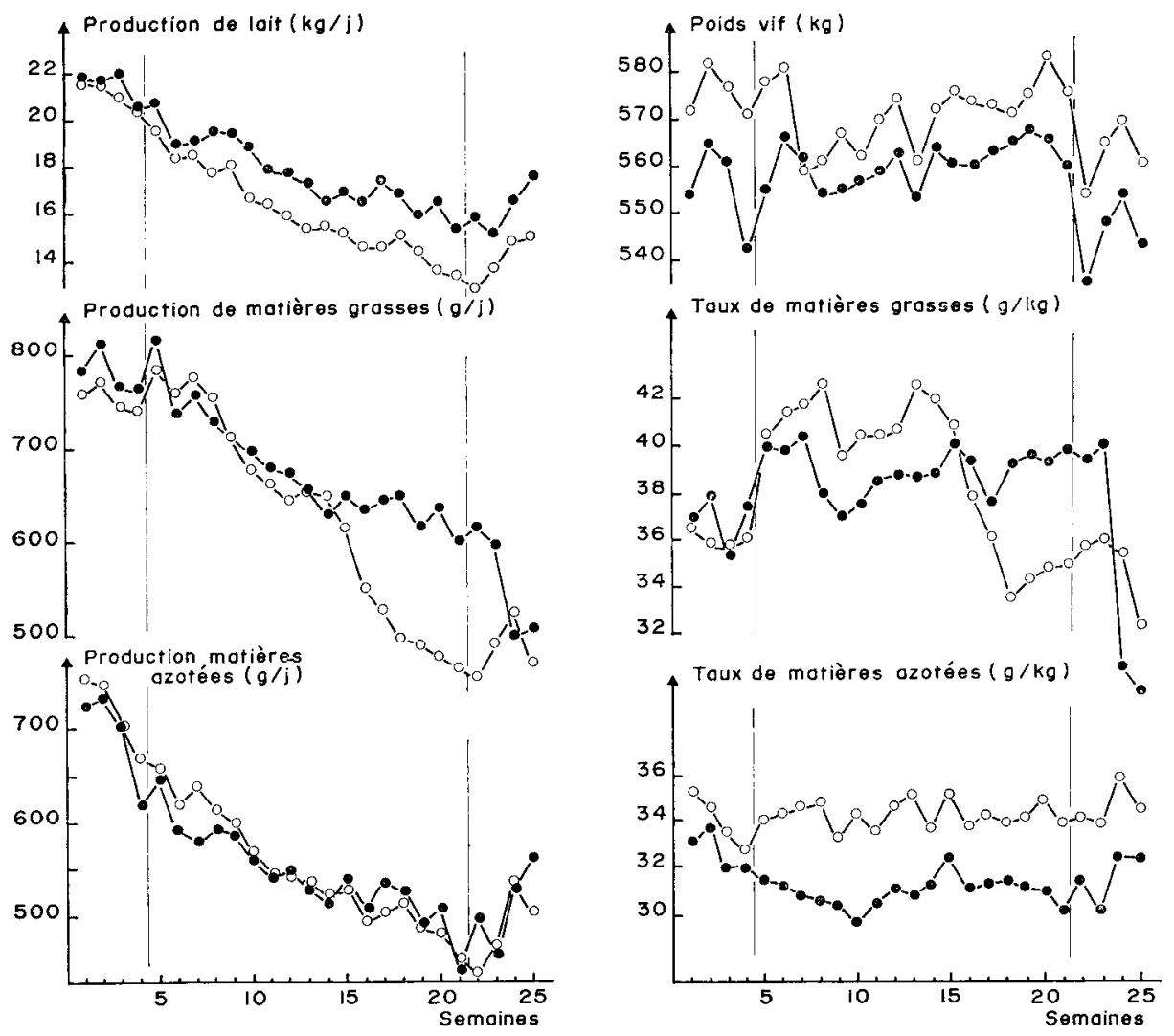

FIG. I. - Évolution des productions et du poids vif au cours de l'expérience

$\mathrm{A}=$ période pré-expérimentale (semaines I et 2 )

- L L L L I (ensilage d'herbe) B $=$ période de transition (semaines 3 et 4 )

$\mathrm{C}=$ période expérimentale (semaine 5 à $2 \mathrm{I}$ )

o___ L Lot II (ensilage de maïs) $\mathrm{D}=$ période de digestibilité (semaine 22)

$\mathrm{E}=$ période post-expérimentale (semaine 23 à 25) 
l'herbe. Cependant, la production de matières azotées a été égale en moyenne et a évolué parallèlement dans les deux lots au cours de l'expérience.

\section{Gain de poids des animaux et variations des quantités de contenus digestifs au cours de l'expérience}

Nous avons estimé le gain de poids vif de chaque animal de 2 façons (tab1. 2 ) ; $\mathrm{I}^{0}$ par la droite de régression des poids hebdomadaires (entre la $3^{\mathrm{e}}$ et la $\mathrm{I} 7^{\mathrm{e}}$ semaine), les deux premières semaines ont été éliminées car les animaux du lot "maïs » recevaient alors l'ensilage à volonté ;

$2^{\circ}$ par différence entre les pesées " triples » effectuées à la $3^{\mathrm{e}}$ et $\mathrm{I} 7^{\mathrm{e}}$ semaine.

Les deux méthodes donnent des résultats concordants et indiquent un gain de poids plus élevé $(\mathrm{P}<0,05)$ pour le lot " maïs ) que pour le lot " herbe " (d'environ Ioo à $\mathrm{I} 80 \mathrm{~g}$ par jour). La quantité de matière sèche totale ingérée par jour a diminué de II p. Ioo et I9 p. Ioo respectivement pour les lots " herbe " et " maiss " entre la $3^{\mathrm{e}}$ et $\mathrm{la} I 7^{\mathrm{e}}$ semaine. On peut donc supposer que les variations de contenus digestifs ont été semblables dans les deux lots et n'ont pas introduit d'erreur dans l'estimation de la différence de gains de poids entre les deux lots.

Nous n'avons pas pu mettre en évidence de différence dans les quantités de contenu digestif du reticulo-rumen entre les deux rations. Nous avons essayé de relier les poids de content1 frais $(y$ en $\mathrm{kg}$ ) à la quantité de matière sèche totale ingérée, à la quantité de matière sèche d'ensilage ingérée, au pourcentage d'aliment concentré et au poids des animaux. La liaison simple la plus étroite a été obtenue avec le poids vif et la liaison double la plus étroite avec le poids vif ( $X_{1}$ en $\mathrm{kg}$ ) et la quantité de MS d'ensilage ingérée $\left(\mathrm{X}_{2}\right.$ en $\left.\mathrm{kg}\right)$.

$$
y=0,200 \mathrm{X}_{1}+3,98 \mathrm{X}_{2}-55,7 \quad r=0,872 \quad \mathrm{P}<0,02
$$

Mais la précision de cette estimation est faible ( $\mathrm{S} y x=\mathrm{r} 0,3)$. Nous n'avons donc pas corrigé les gains de poids des animaux des 2 lots pour tenir compte des variations de contenus digestifs.

\section{Production d'acides gras volatils (AGV)}

Comme le taux butyreux et la composition des matières grasses du lait, la composition du mélange d'AGV du rumen des animaux en lot a été nettement différente entre les 2 lots à la $7^{\mathbf{e}}$ semaine (tab1. 6). Avec l'ensilage de maîs, la proportion d'acide acétique est plus faible $(P<0,05)$ qu'avec l'ensilage d'herbe $(48,6$ contre 56,9 p. IOo); au contraire, celle d'acide butyrique est plus élevée $(27,0 \mathrm{p}$. Ioo contre 19,5 $\mathrm{P}<0,05)$. Par contre, à la $\mathrm{I} 7^{\mathrm{e}}$ semaine, la différence de composition est plus faible, principalement par suite de l'évolution du lot " maïs ». En effet, dans ce lot, la proportion d'acide butyrique a fortement baissé entre la $7^{\mathrm{e}}$ et $1 \mathrm{a} I 7^{\mathrm{e}}$ semaine, tandis que celle de l'acide acétique augmentait. Pour chaque période et dans chaque lot (ou pour les 2 lots réunis), on n'a pas tronvé de liaison entre le taux butyreux et la proportion de chaque acide ou la proportion des acides acétique + butyrique ou le rapport acide acétique/acide propionique. Par contre, pour le lot " maîs », la diminution ( $y$ en $g$ par $\mathrm{kg}$ ) du taux butyreux entre la $7^{\mathrm{e}}$ et la $I 7^{\mathrm{e}}$ semaine est étroitement liée à la diminution ( $x$ en points $\mathrm{p}$. IOo) de la proportion de l'acide butyrique :

$$
y=0,93 x-2,34 \quad r=0,94 \quad \mathrm{P}<0, \mathrm{OI}
$$


Cette liaison est encore plus étroite $(r=0,86-\mathrm{P}<0,00 \mathrm{I})$ si on considère l'ensemble des 2 lots.

L'étude cinétique de la composition des $A G V$ dans le jus de rumen des vaches fistulisées (fig. 2) montre une évolution similaire dans les 2 lots; la teneur en acide

TABLEAU 6

Composition du mélange d'A.G.V. dans le jus de rumen

(moyenne de 6 vaches par lot)

\begin{tabular}{|c|c|c|}
\hline & Lot " herbe" & Lot "maïs" \\
\hline \multicolumn{3}{|l|}{ ze semaine } \\
\hline Acide acćtique $\ldots \ldots \ldots \ldots \ldots$ & 56,9 & 48,6 \\
\hline Acide propionique $\ldots \ldots \ldots \ldots$ & $\geq 1,1$ & 21,0 \\
\hline Acide isobutyrique .......... & 0,5 & 0,1 \\
\hline Acide butyrique $\ldots \ldots \ldots \ldots \ldots$ & 19,5 & 27,0 \\
\hline Acide isovalérianique . . . . . . . . & 0,8 & 0,3 \\
\hline Acide valérianique $\ldots \ldots \ldots \ldots \ldots$ & 1,0 & 2,8 \\
\hline \multicolumn{3}{|l|}{$1 \%$ semaine } \\
\hline Acide acćtique $\ldots \ldots \ldots$ & 51,2 & 52,7 \\
\hline Acide propionique .... & 19,6 & 18,2 \\
\hline Acide isobutyrique $\ldots \ldots \ldots \ldots$ & 0,6 & 0,4 \\
\hline Acide butyrique $\ldots \ldots \ldots \ldots \ldots$ & 22,0 & 23,3 \\
\hline Acide isovalérianique..$\ldots \ldots \ldots$ & 0,9 & $0, t^{\prime}$ \\
\hline Acicle valérianique $\ldots \ldots \ldots \ldots$ & $2, \mathbf{t}^{\prime}$ & 4,8 \\
\hline
\end{tabular}

acétique baisse très fortement (de plus de 15 points) dans les 5 ou 6 heures suivant le début du repas du matin (aliment concentré + betterave déshydratée + ensilage), puis remonte ensuite très progressivement. La teneur en acide butyrique augmente dans le même temps de Io à I 5 points, mais se maintient ensuite à ce niveau pendant une dizaine d'heures avec une légère augmentation après le repas du soir (aliment concentré + ensilage). Les proportions moyennes sur 24 heures obtenues à partir de cette étude cinétique sur vaches fistulisées vont dans le même sens que celles obtenues également avec betteraves déshydratées sur les vaches en lot, la proportion d'acide acétique est plus faible avec l'ensilage de maîs $(59,5$ p. Ioo) qu'avec l'ensilage d'herbe (6r,3 p. I00), par contre, celle d'acide butyrique est plus élevée (I 8,0 contre I6,2 p. IOO).

\section{Corps cétoniques}

Les teneturs du sang en corps cétoniques ( $\mathrm{I} 7^{\mathrm{e}}$ semaine) sont très élevées (probablement en liaison avec la teneur élevée en acide butyrique dans le jus de rumen) pour les 2 lots : 14,7 et $16,8 \mathrm{mg} / \mathrm{I}$ oo $\mathrm{ml}$ de sang respectivement pour les lots "herbe" et " maïs ". Le rapport entre l'acide $\beta$-hydroxybutyrique et la somme acétone + acide acéto-acétique est significativement plus élevé $(\mathrm{P}<0, \mathrm{OI})$ avec le lot "maïs" $(4,73 \pm 0,78)$ qu'avec le lot " herbe " $(2,01+0,52)$. 


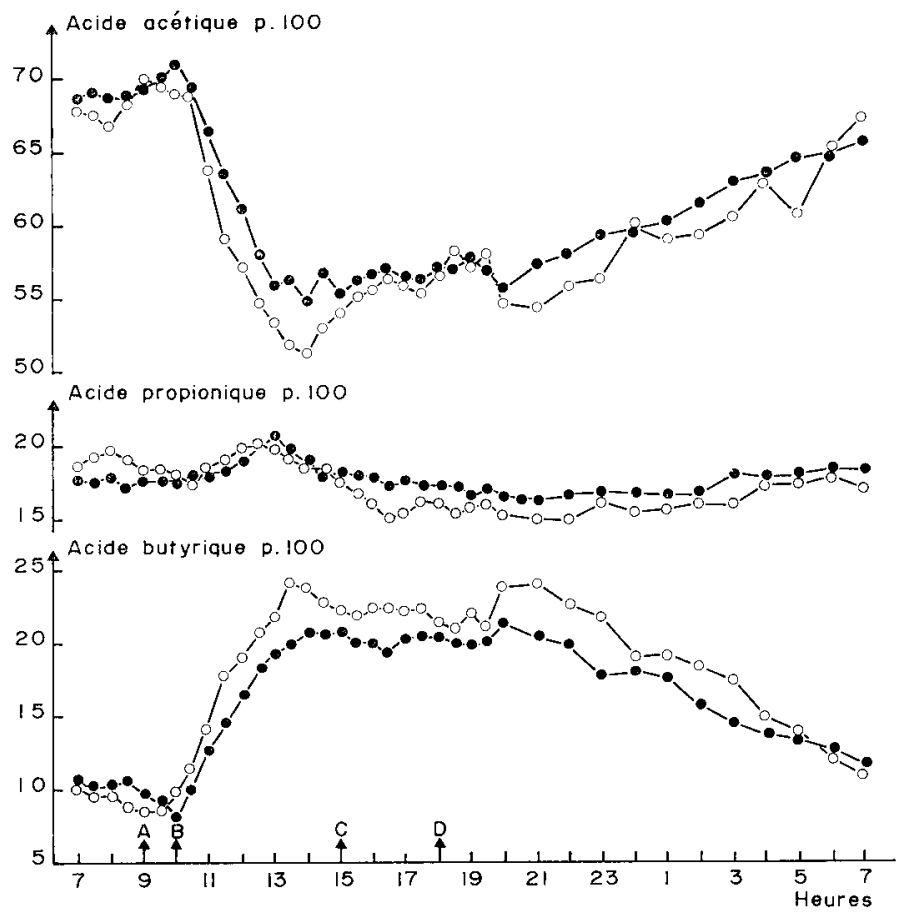

FIG. 2. - Evolution de la composition en acides gras volatils du jus de rumen au cours de la joumée (moyenne de 3 vaches fistulisées)

- - Ensilage d'herbe $\mathrm{A}=$ distribution d'aliment concentré + betteraves déshydratées

$0-\ldots$ Ensilage de naïs $\mathrm{J}=$ distribution d'ensilage

$\mathrm{C}=$ distribution d'aliment concentré

$\mathrm{D}=$ distribution d'ensilage

\section{DISCUSSION}

Pour un apport énergétique identique, la prođuction de lait a été plus élevée avec 1'ensilage d'herbe qu'avec celui de maïs, mais le gain de poids a été significativement plus faible. Les productions moyennes de matières grasses et de matières azotées n'ont pas différé entre les 2 lots.

Cette production plus importante de lait ne correspond pas à une suralimentation relative du lot " herbe " par rapport au lot "maïs ". En effet : Io la suralimentation calculée des 2 lots a été la même ; $2^{\circ}$ l'erreur dans 1'estimation de la teneur en MS des ensilages faite en utilisant une étuve $\left(80^{\circ}\right)$ doit être faible $(<$ à 4 p. Ioo, FatianofF et GoueT, I970) et identique pour les deux ensilages puisque leurs teneurs en corps volatils (AGV et ammoniaque) sont identiques ; $3^{\circ}$ 1'apport énergétique de l'ensilage d'herbe a peut-être été surestimé puisque nous avons utilisé comme valeur énergétique celle obtenue sur moutons $(0,65 \mathrm{UF} / \mathrm{kg} \mathrm{MS})$, alors que celle obtenue à partir des vaches est plus faible $(0,60 \mathrm{UF} / \mathrm{kg} \mathrm{MS}) ; 4^{\circ}$ le taux de protéines du lait a été plus élevé $(\mathrm{P}<0,05)$ avec le lot " maïs ». Or, le taux de protéines augmenterait avec le niveau des apports énergétiques (cf. revues de Rook, I96I et de Aurior, et 
JARRIGE, I962). Cependant, KIRCHGESSNER (I967) fait remarquer que plusieurs auteurs ont enregistré une teneur en protéines faible avec de l'ensilage d'herbe. L'apport azoté, légèrement plus faible dans le lot "maïs ", ne paraît pas non plus être la cause de ces différences de production puisqu'on sait que seule une carence azotée assez prononcée provoque une baisse de la production laitière accompagnée d'une baisse de la production de matières azotées (ce qui n'est pas le cas avec le lot "maïs »). Cet apport azoté était d'ailleurs suffisant pour couvrir les besoins ( ${ }^{1}$ ) azotés.

Il semble donc que le partage de l'énergie disponible, une fois les besoins d'entretien couverts, entre la production du lait et le gain de tissu corporel ait été différent entre les deux rations. La ration " ensilage de maïs " favoriserait le dépôt corporel au détriment de la production de lait au moins comparativement à la ration " ensilage d'herbe ". Cette différence d'orientation dans l'utilisation de l'énergie est à rapprocher de celle provoquée par les régimes à base d'aliments concentrés ou de fourrages broyés (voir introduction) ; elle s'en distingue cependant car c'est la production laitière qui baisse et non la production de matières grasses (au moins dans la première partie de l'expérience). Ceci est probablement à relier à la nature butyrique des fermentations dans le rumen avec l'ensilage de maïs alors qu'elles sont généralement de type propionique avec les régimes précédents. In effet si la proportion d'acide propionique dans le rumen a été la même pour les 2 lots, celle d'acide butyrique et celle d'acide valérianique ont été beaucoup plus élevées dans le lot " maîs " que dans le lot " herbe " (respectivement $+7,5$ et + I,8 points p. Ioo). Il est en outre possible que l'ensilage de maïs (quoique récolté à un stade assez précoce) ait donné naissance à une quantité plus importante de glucose que celui d'herbe puisque une partie de l'amidon de maïs ne serait digérée qu'au niveau de l'intestin (ØRskov et al., I969; THIVEND et JOURNET, r970).

Or on sait (ARMSTRONG et BIAXTER, I957) que le glucose, 1'acide propionique et 1'acide butyrique ont une meilleure efficacité pour l'engraissement que l'acide acétique. BLAXTER (I962), à partir des résultats de BALCH et de ELLIOT et LoOsLI, a montré que le rendement de l'énergie métabolisable pour la production laitière serait maximum pour des teneurs en acide acétique dans le mélange d'AGV du rumen comprises entre 50 et $60 \mathrm{p}$. Ioo. On sait également que l'acide acétique et l'acide butyrique (par l'intermédiaire de l'acide $\beta$-hydroxybutyrique) sont des précurseurs des matières grasses du lait. Il semble d'après différents essais d'infusion d'AGV dans le rumen (Rook BALCH, I96I ; ROoK et al., I965; STORRy et Rook, I965; JORGENSEN et al., I965; WIL,SON et al., I967) que l'acide acétique augmente la production de lait et le taux butyreux alors que l'acide butyrique n'améliore pas ou améliore moins la production laitière, mais augmente plus le taux butyreux.

Deux autres faits semblent indiquer que la mobilisation des réserves corporelles est moins importante dans le lot " maïs ». Il s'agit d'une part de la proportion d'acides gras longs ( < C I8) dans le lait qui est plus faible dans le lot " maïs " et d'autre part du rapport acide $\beta$-hydroxybutyrique/acétone + acide acéto-acétique qui est beaucoup plus élevé $(\mathrm{P}<0, \mathrm{O}$ I) avec l'ensilage de maïs. Or les résultats de HizBiтt et al. (I967 et I969) et de KRONHELD (I970) montrent que ce rapport est beaucoup plus faible chez les vaches acétonémiques qui utilisent leurs réserves corporelles que chez les vaches normales. BJORNTÖRP et SCHERSTEN (I967) ont montré que l'infusion 
d'acide $\beta$-hydroxybutyrique à des chiens avait un effet anti-lipolytique dont l'intensité semblait liée au rapport acide $\beta$-hydroxybutyrique/acide acéto-acétique.

La diminution rapide du taux butyreux dans la deuxième partie de l'expérience avec l'ensilage de maïs et les modifications de composition des matières grasses (diminution de la part des acides gras courts et de l'acide stéarique au profit de celle des acides gras longs insaturés) ressemblent à celles généralement observées avec des régimes à forte proportion d'aliment concentré (GHADAKI, I968), les diminutions du taux butyreux étant alors reliées à une diminution de la proportion d'acide acétique dans le rumen (et à une augmentation de celle de l'acide propionique). Par contre, les baisses de taux butyreux que nous avons enregistrées ici sont très étroitement liées à la diminution de la proportion d'acide butyrique dans le rumen et n'ont pas de rapport avec la teneur en acide propionique ou en acide acétique + butyrique. Il faut remarquer que les proportions d'acide butyrique enregistrées en début d'expérience sont exceptionnellement élevées probablement par suite de la présence de betterave dans la ration. Outre 1'hypothèse d'un effet direct sur le taux butyreux des produits terminaux de la digestion comme précurseurs des matières grasses du lait, il est possible également que ces nutriments aient induit, au niveau du tissu adipeux, une augmentation de l'activité enzymatique de synthèse (OPSTVEDT et al., I967) ou bien qu'au niveau du tissu mammaire l'activité enzymatique de synthèse ait baissé (la diminution des quantités d'acide gras courts sécrétés par kg de lait va dans ce sens) tandis que l'activité des déshydrogénases augmentait comme semble le prouver l'augmentation relative des acides gras mono-insaturés à I 6 ou I 8 atomes de carbone. Il est intéressant de noter un effet d'associativité entre aliments puisque les betteraves déshydratées ont entraîné une baisse du taux butyreux avec l'ensilage de mais mais non avec l'ensilage d'herbe.

D'un point de vue zootechnique, le régime ensilage d'herbe $(24 \mathrm{p}$. Ioo MS) à volonté $+\mathrm{I} 2 \mathrm{~kg}$ de betteraves a assuré en plus des besoins d'entretien une production de 8 à $9 \mathrm{~kg}$ de lait. Si l'ensilage de maïs avait été distribué à volonté, il aurait permis un niveau énergétique de la ration de base plus élevé. En contrepartie, la ration à base d'ensilage de maìs, bien qu'excédentaire en énergie, a permis une moins bonne persistance de la production laitière et un léger engraissement des animaux. Il est nécessaire de confirmer ces résultats en absence de betteraves et avec des maîs à d'autres stades de maturité. Le régime ensilage de maïs seul est probablement un régime " limite " puisqu'une légère modification de la composition de la ration a entraîné une chute importante du taux butyreux, alors que, dans la première partie de l'expérience, celui-ci était nettement supérieur à celui observé avec l'ensilage d'herbe.

$$
\text { Reçu pour publication en février } 1971 .
$$

\section{SUMMARY}

COMPARISON OF THE EFFECT OF MAIZE AND GRASS SILAGE UPON THE MILK PRODUCTION OF DAIRY COWS

The aim of the present trial was to compare the utilization by dairy cows of maize silage with that of pasture grass silage for milk production and to study particularly the effect of these feeds upon the energy metabolism of the animals. 
After a pre-experimental period of 3 weeks, 2 groups of 6 cows received 2 isoenergetic and isonitrogenous diets based one on grass silage ("grass group ") and the other on maize silage (" maize group ") and both containing fresh sugar beet (first ro weeks) or dehydrated sugar beet (last 7 weeks). The amount of concentrates fed to each cow was calculated according to its milk yield during the pre-experimental period and to a theoretical decrease in this yield of I.5 p. Ioo per week. The energy value of each silage was calculated from its digestibility measured by means of a group of 4 sheep and a group of 4 cows (table I).

The cows of the "grass group " produced more milk than that of the " maize group " $(+1.25 \mathrm{~kg}$; $\mathbf{P}<0.05)$ but their liveweight gain was lower ( - I oo or - I 80 g per day depending on the method of estimation ; $\mathrm{P}<0.05)$. The butterfat contents and yields and the protein yields in milk were not significantly different, but the protein content in milk was higher in the " maize group " $(+2.4$ g p. Ioo; $\mathrm{P}<0.05$ ) (table 2 , fig. I).

The interpretation of these results take account of $I$. the accuracy of the estimation of the food supplies, 2. the composition of the mixture of volatile fatty acids in the rumen (the proportion of acetic acid was lower and that of butyric acid higher in the "maize group " than in the "grass group ": 49 p. I00 versus 57 p. Ioo and 27 p. Ioo versus I9 p. Ioo (table 6), 3. the fattty acid composition of the milk fat (table 5), 4 . the amount and nature of the ketone bodies in the blood.

An associative effect between the feeds was observed (table 3 and fig $\mathrm{I}$ ) since the substitution of fresh sugar beet by dehydrated sugar beet resulted, only in the "maize group ", in an important decrease in the butterfat content (-3.4 to $14.4 \mathrm{~g}$ p. I ooo) as well as a change in the fatty acid composition of the milk (table 5). These changes were associated with the decrease in the proportion of butyric acid in the rumen (table 6) whereas that of propionic acid did not change.

\section{RÉFÉRENCES BIBLIOGRAPHIQUES}

Armstrong D. G., Blaxter K. L., I957. The utilization of acetic, propionic and butyric acids by fattening sheep. Brit. J. Nutr., 11, 4 I 3

Auriol P., Jarrige R., ig62. Possibilités de modifier la composition du lait. Fédération internationale de laiterie. Bulletin 1962 , partie II.

Baldwin R. L., Lin H. J., Cheng W., Cabrera R., Ronning V., I969. Enzyme and metabolite levels in mammary and abdominal adipose tissue of lactating dairy cows. J. Dairy Sci., 52, 183-187.

Bjorntorp P., Schersten T., I967. Effect of $\beta$-hydroxybutyrate on lipid metabolism. Am. J.Physiol., 212, 683-687.

BlAXteR K. L., I962. The energy metabolism of mminants. Hutchinson and Co Ltd, London Wr, $329 \mathrm{pp}$.

Breirem K., Ekern A., Homb T., Hvidstin H., Presthegge K., Saue O., Ulyesli O., I96i Adjustements of replacement equivalents by aid of feeding experiments. Proc. 2nd symposium on energy metabolism. Wageningen, 292-306.

Breirem K., I954. "Die Nettoenergie als grundlage der bewertung der futtermittel in Nehring K., Ioo jahre Möckern. Dic bewertung der futterstoffe und andere probleme der tiernährung ». Berlin. Deutsche Akad. der Landwirtschaftswissenchaften, t. II, $97^{-108 .}$

Decaen C., Adda J., I966. Évolution de la sécrétion des acides gras des glycérides du lait de vache au cours de la lactation. XVII Congr. Internat. de laiterie, A, I6I-I $7 \mathrm{I}$.

Demarglilly C., Jarrige R, 1970. The effect of method of forage conservation on digestibility and voluntary intake. Proc. XIth Internat. Grassl. Congr., p. 733.

Fatianoff Nathalie, Gount Ph., 1969. Relation permettant de corriger rapidement et avec précision la matière sèche des ensilages séchés à I'étuve. Ann. Zootech., 18, 407-418

Flat W. P., Mol P. W., Munson A. W., Cooper T., ig69. Energy utilization by high producing dairy cows. 11. Summary of energy balance experiments with lactating Holstein cows in Energy Metabolism of farm animals by Blaxter, Thorbeck-Kielanowski. Proc. 4th Symp., Warsaw 1967, $235^{-25}$ I.

GHADAKI M. B., I968. Influence de quelques facteurs alimentaires sur la sécrélion des principunx acides gras du lait de vache. Thèse de docteur ingénieur de l'Université de Clermont-Ferrand, I4I p.

Hibbit K. G., Barrd G. D., I967. An induced ketosis and its role in the study of primary spontaneous bovine acetonaemia. Vet. Rec., 81, 5 I I -5 I 7 .

Hibirtit K. G., Neinl, D., Radford P., r969. The effect of diet on the incidence of induced ketosis in the lactating dairy cow. Res. Vet. Sici, 10, $245-253$.

Jorgensen N. A., Schultz L. H., BArR G. R., I965. Factors influencing milk fat depression on rations high in concentrates. J. Dairy Sci, 48, IO3 I-I039.

JoRglinsen N. A., Schultz I. H., I965. Ration effects on rumen acids, ketogenesis and milk composition. I. Restricted roughage feeding. J. Dairy Sci., 48, 1040. 
Kirchgessner M., Friesecke H., Koch G., I967. Nutrition and the composition of milk. Crosby Lockwood and Son LTD.

KRoNFELD J). S., I970. Ketone body metabolism, its control, and its implications in pregnancy toxae. mia, acetonaemia, and feeding standards, in lhysiology of digcstion and metabolism in the ruminant by A. T. Phillipson, p. 566-583.

LABADIE P., I968. Utilisation comparée du foin et de l'ensilage d'herbe dans l'alimentation des ruminants. Mémoire ENITA, Bordeaux.

Mrrril W. G., Stack S. T., 1965. Feeding value of perennial forages for dairy cows., a review. Anim. Sci. Mimeo Series, 3. no 3 .

Opstvedt J., Baldwin R. L., Ronving M., I967. Effect of diet upon activities of several enzymes in abdominal adipose and manmary tissues in the lactating dairy cow J. Dairy Sci., 50, ro8-1og.

Ørskov F. R., lilate W. P., MoE P. W., Munson A. W., ig69. The influence of ruminal infusion of volatile fatty acids on milk yield and composition and on energy utilization by lactating cows. Brit. J. Nutr., 23, 443-453.

Orskov E. R., Fraser C., KAY R. N. B, I969. Dietary factors affecting the digestion of starch in the rumen and small and large intestines of early weaned lambs, Brit. J. Nutr., 23, 217-226.

Procos J., I96I. Modifications of the spectrophotometric determination of ketones bodies in blood enabling the total recovery of beta-hydroxybutyric acid. Clin. Chem., 7, 97.

Rigaud J., Journet M., ig7o. Méthode de dosage des acides gras volatils dans le liquide du rumen. Ann. Biol, anim. Bioch. Biophys., 10, I5I-157.

Rook J. A. 1., I96I. Variations in the chemical composition of the milk of the cow. Part I. Dairy Sci. A bstr., 23, $25 \mathrm{I}^{-2} 58$.

Rook J.A. Ii., BALCH C. C., I96I. The effects of intraruminal infusions of acetic, propionic and butyric acids on the yield and composition of the milk of the cow. Brit J.Nutr., 15, 361-369.

Rook J. A. F., I3ALCH C. C., Johnson V. W., I 965 . Further observations on the effects of intraruminal infusions of volatile fatty acids and lactic acid on the yield and composition of the milk of the cow. Brit. J. Nutr., 19, 93-99.

Rucкевusch Y., I963. Recherches sur la régulation centrale du comportement alimentaire chez les ruminants. Thèse Doct. Sci, Lyon.

Snedecor G. W., Cochran W. G., I968. Statistical methods, 6édition.

STORRY J. E., Rook J. A. F., r965. Effect in the cow of intraruminal infusions of volatile fatty acids and of lactic acid on the secretion of the component fatty acids of the milk fat and on the composition of blood. Biochem. J., 96, 2 I0-2I7.

Thivend P., Journet M., I97o. Utilisation digestive de l'amidon de maïs chez le ruminant. Ann Biol. anim. Bioch. Biophys., 10, 323-326.

VAN Soest P. J., I963. Ruminant fat metabolism with particular reference to factors affecting low milk fat and feed efficiency, a review. J. Dairy Sci., 46, 204-2 I6.

Wilson G. F., DAVEY A. W. I', DOLBY R. H., I967. Milk composition as affected by intra-ruminal infusion of volatile fatty acids to cows on a restricted ration. N. Z. J. Agric. Res., 10, 2I 5-225. 